H.P. BONZEL
$1, \infty$
$\mathrm{YU}^{2}$

D.K. YU
M. SCHEFFLER

\title{
The three-dimensional equilibrium crystal shape of Pb: Recent results of theory and experiment
}

\author{
${ }^{1}$ Institut für Bio- und Nanosysteme, IBN-3, Forschungszentrum Jülich, 52425 Jülich, Germany \\ ${ }^{2}$ Fritz-Haber-Institut der Max-Planck-Gesellschaft, Faradayweg 4-6, 14195 Berlin, Germany
}

\section{Received: 16 October 2005/Accepted: 11 December 2006 \\ Published online: 9 March 2007 • () Springer-Verlag 2007}

ABSTRACT The three-dimensional equilibrium crystal shape (ECS) is constructed from a set of 35 orientation-dependent surface energies of fcc $\mathrm{Pb}$ which are calculated by density functional theory in the local-density approximation and distributed over the [110] and [001] zones of the stereographic triangle. Surface relaxation has a pronounced influence on the equilibrium shape. The (111), (100), (110), (211), (221), (411), (665), $(15,1,1),(410)$ and $(320)$ facets are present after relaxation of all considered surfaces, while only the low-index facets (111), (100) and (110) exist for the unrelaxed ECS. The result for the relaxed $\mathrm{Pb}$ crystal state is in support of the experimental ECS of $\mathrm{Pb}$ at $320-350 \mathrm{~K}$. On the other hand, approximating the surface energies of vicinal surfaces by assuming a linear relationship between the $\mathrm{Pb}(111)$ first-principles surface energy and the number of broken bonds of surface atoms leads to a trivial ECS that shows only (111) and (100) facets, with a sixfold symmetric (111) facet instead of the correct threefold symmetry. It is concluded that the broken bond rule in this simple linear form is not a suitable approximation for obtaining the proper three-dimensional ECS and correct step formation energies.

PACS 05.70.Np; 61.50.Jr; 68.35.Md; 71.15.Mb

\section{1}

\section{Introduction}

The surface free energy is a fundamental property of solids and liquids. For crystalline solids it is generally anisotropic, i.e., it depends on the orientation of the surface. In this case the surface free energy is particularly difficult to measure, especially for structurally and chemically well-defined surfaces [1-4]. Hence it is not surprising that many theoretical attempts have been undertaken to generate hopefully trustworthy values of this quantity. Some were empirical, starting from surface free energies of liquids (e.g., metals) and transposing them to the solid state [5,6]. Other semi-empirical studies have been summarized by Galanakis et al. [7]. More recently, full potential first principles calculations have produced surface energies of many crystalline materials [7-18] mostly for low-index orientations. Although there can be considerable scatter in theoretical values for a single material and orientation [16, 19], it has been suggested by

Fax: +49 246161 2950, E-mail: h.bonzel@fz-juelich.de some authors that the lack of experimental surface energy data for well-defined orientations may be replaced by ab initio calculations $[15,17,20]$.

Theoretical attempts of describing the more or less complete anisotropy of the surface free energy of crystals by invoking attractive pairwise atomic interaction potentials have been numerous, beginning in 1931 with the work by Stranski et al. [21-26] followed by others [27-30]. A different phenomenological description of the anisotropy and to some extent of the temperature dependence of the surface free energy was based on the terrace-step-kink model of a crystalline surface [31] assuming the existence of steps of monoatomic height, their thermal formation, interaction and roughening $[32,33]$. The most important result of this work and of later additions [34-38] is the following equation:

$f(T, \theta)=f_{0}(T)+f_{1}(T) \tan \theta+f_{3}(T) \tan ^{3} \theta$

Here $f(T, \theta)$ is the surface free energy per unit area for a particular polar angle $\theta$ relative to a stepless low-index orientation, $\tan \theta=\mathrm{d} z / \mathrm{d} x$ the density of monatomic steps, with $z(x)$ being a one-dimensional shape function, $f_{0}(T)$ the surface free energy of a flat terrace, $f_{1}(T)$ the free formation energy of an isolated step, and $f_{3}(T)$ a step-step interaction energy, respectively. This equation is valid for a finite range of orientation. A long range step-step interaction proportional to $\tan ^{2} \theta$ is not included because so far the evidence for this term is controversial [39-44]. Configurational and vibrational entropies govern the temperature dependence of all the free energies in (1). In three dimensions, the anisotropic surface free energy is visualized as a polar plot of $\gamma(\boldsymbol{n})$ where the unit vector $\boldsymbol{n}$ represents a crystallographic direction (surface orientation). Alternatively, this quantity may be written as $\gamma(\theta, \varphi)$, where $\varphi$ is an azimuthal angle. For simplicity we consider one-dimensional azimuthal cuts and note that $\gamma(\theta)=$ $f(\theta) \cos \theta[35]$.

An important issue in connection with the anisotropic surface free energy $\gamma(\boldsymbol{n})$ is the question of the equilibrium shape of a crystal, i.e., the shape of an isolated crystallite in the state of thermal equilibrium. The classic solution to this problem, which has its own interesting history [27], is the well known graphical Wulff construction [45]. The rigorous proof of this construction was given by Dinghas [46]. A mathematical analogue to the Wulff construction is the Legendre 
transform [47]. It is convenient to use a computer code called Wulffman [48] that generates the three-dimensional equilibrium crystal shape (ECS) from any reasonable set of $\gamma(\boldsymbol{n})$ values. On the other hand, an experimental ECS of a micronsized crystallite which may be imaged at elevated temperature by electron microscopy, is an important source of information on $\gamma(\boldsymbol{n})$. This image (or parts of it) may be converted via an inverse Wulff construction (or Legendre transform) to yield portions of the relative anisotropy of the surface free energy [42, 49-52]. Furthermore, it was recently shown that, in principle, absolute surface free energies can be obtained by investigating the temperature variation of particular facets on an experimental ECS $[19,53]$. Hence the equivalence of the anisotropic gamma-plot $\gamma(\boldsymbol{n})$ and the ECS is important for deriving absolute surface free energies of certain low-index orientations [53] and the complete relative surface energy anisotropy [54]. In this paper we will demonstrate the use of the Wulff construction to convert recent theoretical surface energy data of $\mathrm{Pb}[16,18,55]$, which were calculated for a range of orientations, into the corresponding equilibrium shapes. It will become obvious that a high level of accuracy in $\gamma(\boldsymbol{n})$ is needed to generate realistic crystal shapes [55] and to determine reliable step formation energies according to (1).

\section{Orientation dependent surface energies and ECS of $\mathbf{P b}$ by first principles}

First principles surface energies of $\mathrm{Pb}$ have been reported for low-index orientations $[7,8,13,14,16,56,57]$ and for vicinal orientations $[7,18,55]$. The most extensive set of 35 theoretical surface energies of vicinal and lowindex orientations, in their unrelaxed as well as fully relaxed state, was obtained by $\mathrm{Yu}$ et al. using density-functional theory (DFT) [18]. The approach is based on ab initio, normconserving pseudo potentials. Relativistic effects important for $\mathrm{Pb}$ are included at the scalar relativistic level while the spin-orbit coupling terms are averaged. The exchangecorrelation interaction is described within the local-density or the general gradient approximation. The convergence of the results was checked as a function of plane-wave cutoff, $\boldsymbol{k}$ mesh, vacuum and slab thickness, and the effect of nonlinearity of the core valence exchange-correlation interaction. The numerical error bar for the surface energies relative to each other is estimated at $< \pm 0.1 \mathrm{meV} / \mathrm{A}^{2}$. Further details are given in [58] and [16].

Using the data by $\mathrm{Yu}$ et al. $[16,18]$, we investigated the stability of vicinal surfaces with respect to faceting and a first study of sections of the ECS was carried out by graphically constructing facets from polar plots of theoretical $\gamma(\theta)$ values in the major [110] and [001] zones of the crystallographic triangle [55]. It was found that surface relaxation has a considerable influence on surface stability and the ECS. However, the primary objective of this previous work was to check the validity of (1) on the basis of first principles surface energies. In particular, a linear range of $f(\theta)$ versus step density $\tan \theta$ relative to the (111) orientation was expected, and furthermore, it was of interest to find out for which range of misorientation linearity would hold. The latter point would be a direct consequence of the magnitude of the step-step interaction energy relative to the step formation energy.
The results for relaxed surfaces, displayed for $\mathrm{Pb}(111)$ vicinal A and B stepped surfaces in Fig. 1, show an excellent linear behavior up to $\tan \theta=0.22\left(\theta \leq 12^{\circ}\right)$ and thus allow the evaluation of theoretical step formation energies which are 158 and $139 \mathrm{meV}$ for A and B steps, respectively. It was concluded that first principles theory confirms the simple form of (1), which is a result of statistical thermodynamics applied to the terrace-step-kink model of surfaces [34, 37, 38]. Hence theoretical surface energies of vicinal surfaces, obtained for a particular zone (i.e., one kind of step) in conjunction with (1), are a reliable way to extract step formation energies.

Galanakis et al. pointed out that theoretical surface energies $\gamma(\theta)$, when converted to units of energy per surface atom, $F(h k l)$, are an almost perfect linear function of the number of broken bonds at the surface, $N_{\mathrm{bb}}(h k l)$ (per unit cell of surface) $[7,15]$. Here, Miller indices $h k l$ define the surface orientation $\boldsymbol{n}$. The larger the step separation ( $\propto$ unit cell), the larger the number of broken surface bonds. A corresponding plot of $F(h k l)=\gamma(\theta) A(h k l)$, where $A(h k l)$ is the area of the unit cell in $\AA^{2}$, versus $N_{\mathrm{bb}}(h k l)$ is shown in Fig. 2 for the un-

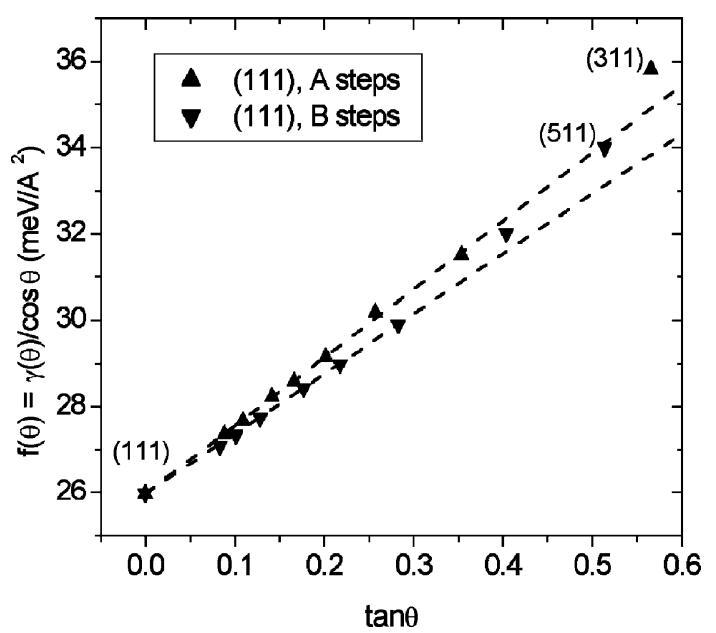

FIGURE 1 Plot of projected surface energy, $f(\theta)$, versus step density, $\tan \theta$, for relaxed $\mathrm{Pb}$ surfaces in the (a) $[01 \overline{1}]$ and (b) [110] zones, representing the inequivalent (111) vicinal $\mathrm{A}$ and $\mathrm{B}$ steps. The lines are linear fits to points at $\tan \theta<0.23$

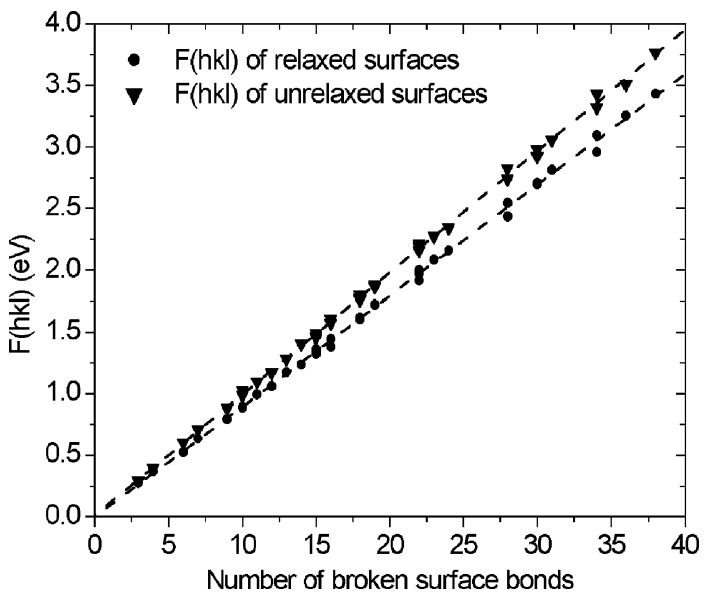

FIGURE 2 Theoretical surface energies $F(h k l)$ in $[\mathrm{eV}]$ per surface atom versus the number of surface bonds which are broken in forming an $(h k l)$ surface. The results of DFT calculations are shown for relaxed and unrelaxed $\mathrm{Pb}$ surfaces [18] 
relaxed and relaxed surfaces of $\mathrm{Pb}$ including all 35 calculated surface energies for each category [18]. The average energy per surface bond is $98.6 \mathrm{meV}$ and $90.0 \mathrm{meV}$ for unrelaxed and relaxed surfaces, respectively. We note that the approximate linearity between surface energy and the number of broken surface bonds in Fig. 2 is present even for $\mathrm{Pb}$, which is not a transition metal. Also, $F(h k l)$ versus $N_{\mathrm{bb}}(h k l)$ is not a unique linear function since several energy values appear for the same $N_{\mathrm{bb}}(h k l)$ in some cases. It will become obvious below that the deviations from linearity are essential for a correct description of the ECS and physical surface properties.

The three-dimensional ECS of $\mathrm{Pb}$ produced from the theoretical surface energies by Wulffman [48] are presented in Fig. 3a, b and c. There are very substantial differences for the unrelaxed and relaxed surfaces. In the case of relaxed surfaces, Fig. 3a, the ECS shows numerous facets besides the expected main low-index facets of (111) (100) and (110) orientations. The (111) facet is threefold symmetric, consistent with unequal A and B step energies, while the (100) facet is fourfold symmetric and the (110) twofold symmetric with a very high anisotropy. The additional facets can be identified as (665) and (221) along the [0 $\overline{1} 1]$ zone, located between (111) and (110) facets. Along the [ $[\overline{1} 10]$ zone the (211), a narrow $(411)$ and a $(15,1,1)$ facet are observed, all located between (111) and (100) facets. Furthermore, in the [100] zone a (320) facet and a very small (410) facet (too small to be visible in Fig. 3a) are present. Although the (410) may be an artifact due to the point like $\gamma(\boldsymbol{n})$ plot, all of the other facets are stable with respect to faceting into (111) and (100) orientations [55]. Other vicinal surfaces are unstable and expected to decompose into neighboring stable orientations [55]. The ECS of Fig. 3a is completely different from an earlier theoretical determination of the ECS of $\mathrm{Pb}$ using first-principles data of low-index orientations [8] together with a cluster energy expansion approach [59]. Although this approach should also support facets other than (111), (100) and (110), none were found for $\mathrm{Pb}$.

In the case of unrelaxed surfaces, the ECS is quite simple and contains just the low-index facets (111), (100) and (110) (black line), as seen in Fig. 3b. This is consistent with the instability of all vicinal unrelaxed surfaces with regard to decomposing into (111) and (100) orientations [55]. The ECS of unrelaxed surfaces is also similar to any ECS calculated on the basis of nearest and next-nearest neighbor interactions $[37,38,60]$. Only further neighbor interactions will introduce new facets, a result which was predicted by Landau prior to 1950 [37]. However, the threefold symmetry of the (111) facet persists for the unrelaxed ECS. The comparison of Fig. 3a and b illustrates the enormous influence of surface relaxation on the ECS. First principles theory includes many body interactions but their influence on surface structure and energy becomes fully effective when surface relaxation is allowed, associated with a smoothing of surface electron charge density [61] and a rearrangement in the positions of near surface atoms [62].

In the general context of the broken bond model mentioned above it has been suggested that surface energies of vicinal orientations may not have to be calculated but instead be estimated by assuming a strict linear relationship between values calculated for low-index surfaces and the number of broken bonds for any vicinal surface [17]. However, this degree of simplification is most likely leading to unreliable results when it comes to a description of properties of vicinal surfaces $[9,63]$. We have recently pointed out [18] that the assumption of a strict broken bond model leads to the wrong ECS as well as erroneous values of step energies. The ECS of $\mathrm{Pb}$ in Fig. 3c, for example, is constructed from the idealized broken bond data. It is similar to that for the unreconstructed case in Fig. 3b but exhibits only (111) and (100) facets. A closer analysis of $\gamma(\boldsymbol{n})$ shows that all vicinal surface orientations on the ECS are stable with respect to faceting into low-index orientations. In the language of Herring [27], this means that all round portions of the gamma-plot $\gamma(\boldsymbol{n})$ correspond to spheres (circles in two dimensions) going through the origin (the Wulff point) and the low-index (111) and (100) orientations. For comparison, the $\gamma(\boldsymbol{n})$ plot calculated for unrelaxed surfaces lies outside these spheres, that for the relaxed surfaces is located inside the spheres. The second difference between Fig. 3b and c is the symmetry of the (111) facet, which is sixfold instead of threefold.

A comparison of the projected surface energies calculated for relaxed surfaces and for the ideal surface bond breaking model in the [ $\left.\begin{array}{lll}1 & 1 & 0\end{array}\right]$ zone, both matched at the (111) orientation, is shown in Fig. 4. The initial slopes, $\mathrm{d} f(\theta) / \mathrm{d} \tan (\theta)$, which equal the step energy in the same units as $f(\theta)$, can be evaluated for both the A and B step regions of vicinal surfaces. Whereas the $f(\theta)$ values calculated by DFT yield $f_{1 \mathrm{~A}}(0)=$ $15.8 \mathrm{meV} / \AA^{2}$ and $f_{1 \mathrm{~B}}(0)=13.9 \mathrm{meV} / \AA^{2}$, the broken bond model data are higher, namely at $f_{1}(0)=18.4 \mathrm{meV} / \AA^{2}$ for both types of steps. In the approximation of the broken bond model there is no difference in A and B step energies, hence the (111) facet is sixfold symmetric (Fig. 3c). This feature is

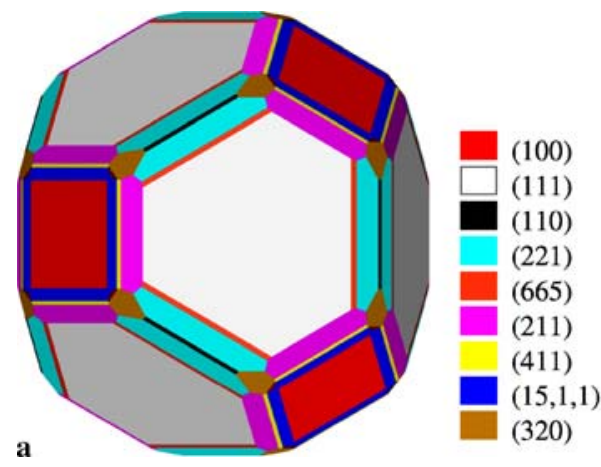

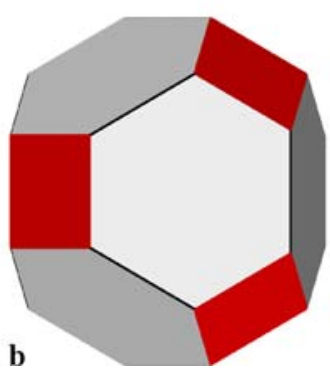

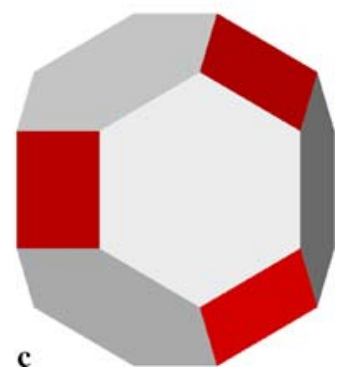

FIGURE 3 Three-dimensional equilibrium crystal shapes of $\mathrm{Pb}$ constructed from the first principles surface energies. (a) Relaxed surfaces; (b) unrelaxed surfaces; (c) with vicinal surface energies according to the broken bond model. Note in (a) and (b) the (110) facet as a black line between neighboring (221) facets. Also note the different symmetry of the (111) facet in (b) (threefold) and (c) (sixfold) 


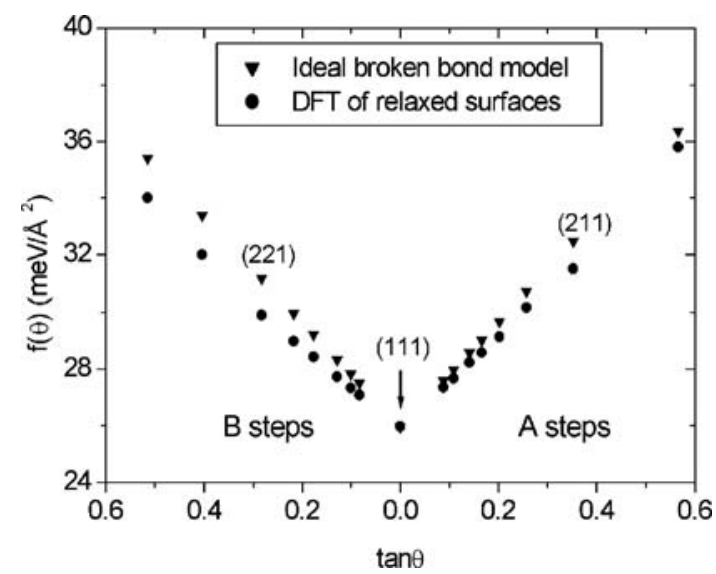

FIGURE 4 Comparison of surface energy, $f(\theta)$, versus step density, $\tan \theta$, for relaxed $\mathrm{Pb}$ surfaces in the [011] and [110] zones, corresponding to $\mathrm{A}$ and $\mathrm{B}$ steps vicinal to $\mathrm{Pb}(111)$, respectively. Two sets of data are shown: one calculated according to the ideal broken bond model, the other for the exact DFT values [18]. Both sets are matched at the (111) orientation

characteristic for the approximation of nearest neighbor interactions, in stark contrast to the $f_{1}(T)$ values calculated by DFT for the unrelaxed surfaces which yield unequal A and B step energies. This comparison shows that accurate theoretical values of all surface energies, including those for vicinal orientations, are needed to obtain realistic step energies as well as the proper ECS of 3D crystallites. The same requirement needs to be fulfilled for the calculation of step-step interaction energies.

\section{Experimental results of the equilibrium crystal shape of Pb imaged by STM}

Scanning electron tunneling microscopy (STM) studies of $\mathrm{Pb}$ crystallites under ultra-high vacuum conditions have proven to be advantageous because of the atomic resolution of the technique [64]. Images show the step-resolved, exact location of facets and permit the analysis of facet shapes as well as the shape of rounded vicinal portions near facets. In general, more quantitative evaluations of equilibrated crystallites are possible than before. To prepare crystallites, $\mathrm{Pb}$ is vapor deposited as a thin film on $\mathrm{Cu}(111)$ or $\mathrm{Ru}(001)$ single crystals, the film is dewet to make isolated particles which are subsequently solidified and equilibrated at a constant temperature $[52,65,66]$. Due to the $\mathrm{Pb} /$ substrate interface, the $\mathrm{Pb}$ crystallites exhibit a truncated crystal shape [28], with a central (111) facet parallel to the close-packed substrate surface. It is important for all quantitative work to image crystallites at the temperature of equilibration to maintain the equilibrium state. Imaging at lower temperatures than the one used to equilibrate the crystallite will change the crystallite's shape and lead to erroneous results $[43,64]$. An ensemble of prepared $\mathrm{Pb}$ crystallites on $\mathrm{Ru}(001)$ imaged by scanning electron microscopy is presented in Fig. 5a which shows also the tip of the STM [67]. A high resolution STM image of a single $\mathrm{Pb}$ crystallite with a diameter of about $1 \mu \mathrm{m}$ and equilibrated at $353 \mathrm{~K}$ is shown in Fig. 5b. A close-up view of a (111) facet with neighboring (112) and (221) facets is presented in Fig. 5c [43].

Because of numerous publications dealing with various aspects of $\mathrm{Pb}$ equilibrium crystal shapes (ECS) [19, 43, 44, 64, 68-77] we will provide here just a brief summary of the results from this work.

- STM images of Pb crystallites show atomically flat (111) facets, which are threefold symmetric, Fig. 5c, consistent with the symmetry of the fcc lattice [68]. The (111) facets are bound by rounded A and B steps where A steps exhibit a (100) ledge and B steps a (111) ledge, respectively. The B step is less rounded than the A step. Single steps of monatomic height, beginning at (screw) dislocation emergence points, are detected within the (111) facet area in some cases $[44,64]$. These steps are essential for efficient equilibration of the crystallites [43, 78, 79].

- The transition between (111) facet and vicinal (rounded) regions is continuous, in agreement with earlier work [41]. The shape function near the (111) facet is characterized by a critical exponent $3 / 2$, which is consistent with the dipole nature of steps and a $1 / x^{2}$ step-step interaction potential [75]. Imaging the PB ECS at elevated temperature is essential for this result.

- A detailed analysis of vicinal shapes near (111) facet boundaries yields elastic step-step interaction energies of 8-10 meV/ $\AA^{2}[43,64]$.

- Absolute step free energies and kink formation energies of A and B steps were obtained from analyzing the temperature dependence of (111) oriented facets on 3D crystallites and of $2 \mathrm{D}$ islands on flat $\mathrm{Pb}(111)$ substrates $[74,76]$. The values for the step energies (at $T=0 \mathrm{~K}$ ) are $128 \mathrm{meV}$ and

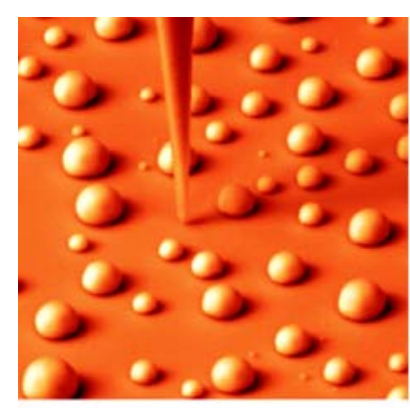

a

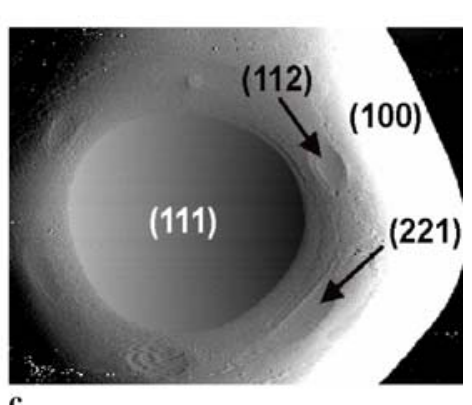

c

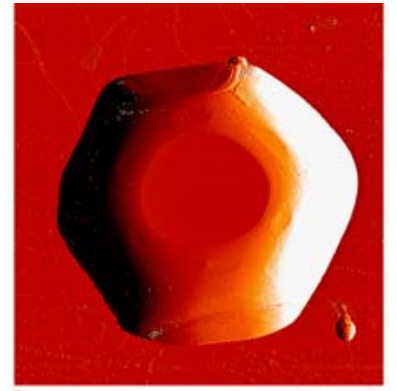

b

(a) Scanning electron microscope image of $\mathrm{Pb}$ particles on a $\mathrm{Ru}(001)$ surface together with an STM tip in tunneling contact. Image size: 25

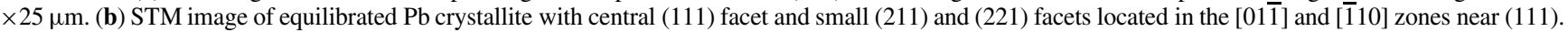
Temperature of equilibration is $353 \mathrm{~K}$. Image size: $1500 \mathrm{~nm} \times 1500 \mathrm{~nm}$. (c) Partial STM image of (111) facet and surrounding twofold symmetric (221) and (211) facets as well as a (100) facet at the contact edge to the substrate. Temperature of equilibration and imaging is $353 \mathrm{~K}$. Image size: ca. $970 \mathrm{~nm} \times 750 \mathrm{~nm}$ 
$116 \mathrm{meV}$ for A and B steps, respectively. The corresponding kink energies are 42.5 and $60.6 \mathrm{meV}$, respectively.

- The combination of quantitative $\mathrm{Pb}$ ECS geometric data and absolute step energies led to the absolute surface energy of $\mathrm{Pb}(111)$ of $27.5 \mathrm{meV} / \AA^{2}$. Values of other orientations, such as (100), (221), (211) and (311) were derived [19]. The ECS equilibrated at 323-353 K shows stable (221) and (211) facets near the (111) facet. The (221) facet can be observed at $T<373 \mathrm{~K}$ while the (211) facet has a somewhat larger range of existence at $T<$ $393 \mathrm{~K}[55]$.

\section{Comparison of theory and experiment}

In this section we compare the results available from first principles theory with those of recent experiments for $\mathrm{Pb}$. First, theoretical surface energies (at $T=0 \mathrm{~K}$ ) of low-index and some vicinal surface orientations are compared with experimental surface free energies obtained at $T=323-353 \mathrm{~K}$ [19] in Fig. 6. It is obvious that there is a considerable spread in the theoretical data which is partly due to different conditions. For example, the results by Galanakis et al. were obtained for unrelaxed $\mathrm{Pb}$ surfaces [7]. The surface energies calculated by Vitos et al. [13] and Feibelman [14, 56] used density functional theory in the general gradient approximation (GGA) while Mansfield and Needs [8] as well as Yu et al. $[16,18]$ used DFT in the local density approximation (LDA). Surface energies for $\mathrm{Pb}$ and other metals calculated with GGA are about 30\% lower than those with LDA [16, 20]. The LDA yields better agreement with experiment, which is believed to be due to a better error cancellation of the surface exchange and correlation energy [57,80-82]. Second, an important difference between theory and experiment in Fig. 6 is the temperature. The surface free energy decreases nonlinearly with increasing temperature due to vibrational and configurational entropy whereby the rate of decrease depends on surface orientation. The smoother the surface, the lower the decrease in $\gamma(\theta)$ with temperature, an effect which was demonstrated for $\mathrm{Pb}$ in the range $470-570 \mathrm{~K}$ [51]. The rate of decrease becomes very low at the low temperature end. The lowest decrease is expected for the (111) orientation although no experimental data are available. Hence one may

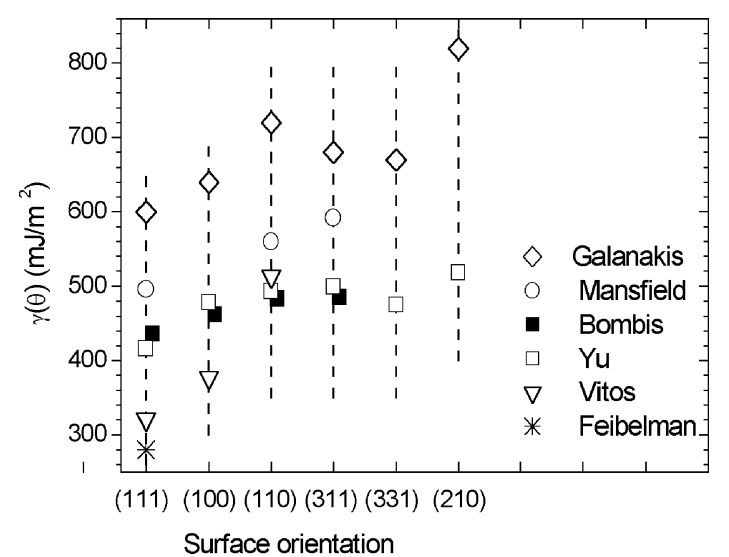

FIGURE 6 Survey of several first principles theoretical Pb surface energies and recent experimental results for six different surface orientations [44]. For references see text argue that the agreement between the theoretical values by $\mathrm{Yu}$ et al. [18] and the experiment at $323-353 \mathrm{~K}$ is very reasonable. The agreement for the other orientations in Fig. 6 would qualitatively improve if a correction for the different temperatures could be made. Other experimental studies of $\mathrm{Pb}$ surface free energies, which have claimed to represent the (111) orientation, report values close to $570-600 \mathrm{~mJ} / \mathrm{m}^{2}$ but these high values are more likely due to vicinal or ill-defined rough surfaces $[83,84]$.

The theoretical step energies for vicinal $\mathrm{Pb}(111)$ surfaces which were calculated in conjunction with the DFT surface energies were also rather different depending on the GGA versus LDA. Feibelman employing the GGA reported 95 and $78 \mathrm{meV}$ for A and B steps [14] while Yu et al. found 158 and $139 \mathrm{meV}$ based on the LDA [18]. The corresponding experimental values are $128 \mathrm{meV}$ and $116 \mathrm{meV}$, respectively [76]. It was concluded that LDA should be preferred because of the same arguments put forward for the theoretical surface energies $[16,20,57,85]$. The fact that the experimental values are lower than the LDA results was rationalized by the non-unique fitting of primary experimental data with Ising type theory [86] to extract the step and kink energies at $T=0 \mathrm{~K}$ [18]. At this point it should be noted that the step energy of $184 \mathrm{meV}$, which was evaluated above according to the broken bond model is even further away from the experimental step energies, not to mention that this model does not distinguish A and B steps.

An analogous situation exists for step energies of vicinal $\mathrm{Cu}(111)$ surfaces [17]. We evaluated the $\mathrm{Cu}$ first principles surface energies in the $\langle 110\rangle$ zone according to (1) and obtained excellent straight lines whose slopes correspond to the formation energies of $\mathrm{A}$ and $\mathrm{B}$ steps of $\mathrm{Cu}(111)$. These are 281 and $269 \mathrm{meV}$, respectively, and predict a small anisotropy of 0.96 at $0 \mathrm{~K}$. These values are in very good agreement with experiment which quotes $262-270 \mathrm{meV}$, depending on the investigative technique [87]. Earlier experiments found $310 \mathrm{meV}$ [88] and $230 \mathrm{meV}$ [89] for the same quantity. There is no reported anisotropy of A/B step energies in the experiments. The step energies by Da Silva et al. agree also well with the first principles results by Feibelman [90] who quotes 270 and $260 \mathrm{meV}$ for A and B steps, respectively. Applying the bond breaking model to $\mathrm{Cu}(111)$ vicinal surface energies and calculating the step formation energy from those leads again to a larger value of $313 \mathrm{meV}$ for both steps. Thus the error introduced by this model is $11 \%-16 \%$ relative to the first principles approach.

Finally, a comparison of the experimental ECS of $\mathrm{Pb}$ with the theoretical ECS, obtained via the Wulff construction from first principles surface energies, shows clearly that a high level of theory is needed to obtain qualitative agreement beyond just the existence of the main low-index facets (111), (100) and (110). The simple pairwise interaction potential [27] or the broken bond model already yield the main facets but a number of additional smaller facets, mostly of twofold symmetry, are predicted by first principles, such as seen in Fig. 3a. Indeed, experimental STM images have revealed at least some of these facets, such as (221) and (211) [19,55], as they are seen in Fig. 5b and c. This is due to several reasons, such as the high resolution of the STM, the smaller size of the crystallites, and the lower temperature of equilibration compared 
to earlier studies $[51,52,65,91-93]$. The (221) and (211) facets are only 16 and 19 degrees away from the central (111) facet which makes them easy to image. Other possible facets such as (320), (411) or (110) are at higher angles relative to (111) and thus in regions of higher slope which are increasingly more difficult to image by the STM tip. The (110) facet in Fig. 3a is quite narrow and highly anisotropic. The step formation energy of the [001] oriented step is very high but will rapidly decrease at $T>0$ because its kink formation energy is near zero. Hence the facet is likely to be unobservable at $T>0$ on the ECS but may be visible on the growth form of $\mathrm{Pb}$ [94]. A similar argument holds for the (411) facet. The predicted (665) facet at $4.76^{\circ}$ misorientation relative to (111), Fig. 3a, is questionable because it represents the first calculated $\gamma(\theta)$ point in that zone which may artificially produce a facet. In view of the large step separation of about $34 \AA$ on that surface the formation of a stable facet seems also rather unlikely. The same argument can be put forward for the $(15,1,1)$ facet next to (100) where the step separation is $26 \AA$. Additional $\gamma(\theta)$ point calculations for vicinal surfaces with even wider terraces are needed to verify (or eliminate) the existence of these facets (very costly calculations).

Experimentally, there is no doubt that even smaller $\mathrm{Pb}$ crystallites could be prepared and equilibrated, even below room temperature, to look for the existence of all theoretically predicted facets and to analyze their temperature behavior. Also, crystallites with a (100) facet parallel to the substrate surface would be advantageous for imaging other regions of the crystallite with high resolution. The only disturbing point is the interaction of the tunneling tip with the $\mathrm{Pb}$ surface which causes occasional tip-surface contacts $[95,96]$ and thus destroys the imaging quality. Hopefully more high resolution scanning probe imaging studies of the ECS of $\mathrm{Pb}$ and other materials will be performed in the future to utilize the great potential of this general approach for getting fundamental surface energetic as well as kinetic data [73,97-99]. Finally, a thorough experimental and theoretical study of supported nanocrystals is very important because the interfacial energy between crystallite and support causes elastic distortions at the interface and thus influences the equilibrium shape of the truncated crystallite $[100,101]$. This problem is particularly relevant in relation to the physics and preparation of quantum dots [102-104]. Surface and interface relaxations will most likely be equally significant here as they are for mesoscopic crystallites.

\section{5}

\section{Conclusions}

- Recent DFT calculated surface energies of $\mathrm{Pb}$ agree well with experimental results obtained at 320-350 K if the effect of temperature is qualitatively taken into account.

- Step energies evaluated from the theoretical surface energies are close to experimental values determined from facet and island shape variation over a large temperature range.

- The experimentally observed threefold symmetry of the (111) facet is confirmed by theory.

- The 3D theoretical ECS of a Pb crystal in its relaxed state exhibits a large number of facets while the ECS in the unrelaxed state shows only (111), (100) and (110) facets. The former ECS is in qualitative agreement with experiment at elevated temperature.

- Theoretical Pb surface energies of 35 orientations, in units of energy per surface atom, show an approximate linear dependence with the number of broken surface bonds.

- Assuming an ideal linear dependence of surface energies with the number of broken bonds results in incorrect crystal shapes and step formation energies.

ACKNOWLEDGEMENTS Special thanks are due to Wolfram Zieger (FHI Berlin) for providing the 3D images of the equilibrium crystal shapes generated by the Wulffman program.

\section{REFERENCES}

1 E.D. Hondros, Proc. R. Soc. London A 286, 479 (1965)

2 E.D. Hondros, M. McLean, "Structure et Propriétés des Surfaces des Solides", presented at Colloques Intern. du CNRS, Paris (1969)

3 E.D. Hondros, Surface Energy Measurements, in Physicochemical Measurements in Metals Research, Techniques of Metals Research, ed. by R.A. Rapp (Interscience, New York, 1970), vol. IV, part 2, pp. 293 348

4 D. Sander, H. Ibach, Surface Free Energy and Surface Stress, in Physics of Covered Solid Surfaces, Vol. III-42-A2, (Landolt-Börnstein), ed. by H.P. Bonzel, New Series N Edn. (Springer, Berlin, 2002), vol. III-42A2, pp. 4.4-1-49

5 A.R. Miedema, Z. Metallkd. 69, 287 (1978)

6 A.R. Miedema, R. Boom, Z. Metallkd. 69, 183 (1978)

7 I. Galanakis, N. Papanikolaou, P.H. Dederichs, Surf. Sci. 511, 1 (2002)

8 M. Mansfield, R.J. Needs, Phys. Rev. B 43, 8829 (1991)

9 M. Methfessel, D. Henning, M. Scheffler, Appl. Phys. A 55, 442 (1992)

10 M. Methfessel, D. Henning, M. Scheffler, Phys. Rev. B 46, 4816 (1992)

11 H.L. Skriver, N.M. Rosengaard, Phys. Rev. B 46, 7157 (1992)

12 P. Feibelman, Phys. Rev. B 52, 16845 (1995)

13 L. Vitos, A.V. Ruban, H.L. Skriver, J. Kollár, Surf. Sci. 411, 186 (1998)

14 P. Feibelman, Phys. Rev. B 62, 17020 (2000)

15 I. Galanakis, G. Bihlmayer, V. Bellini, N. Papanikolaou, R. Zeller, S. Blügel, P.H. Dederichs, Eur. Phys. Lett. 58, 751 (2002)

16 D. Yu, M. Scheffler, Phys. Rev. B 70, 155417 (2004)

17 J.L.F. Da Silva, C. Barreteau, K. Schroeder, S. Blügel, Phys. Rev. B 73, $125402(2006)$

18 D. Yu, H.P. Bonzel, M. Scheffler, Phys. Rev. B 74, 115408 (2006)

19 C. Bombis, A. Emundts, M. Nowicki, H.P. Bonzel, Surf. Sci. 511, 83 (2002)

20 J.L.F. Da Silva, C. Stampfl, M. Scheffler, Surf. Sci. 600, 703 (2006)

21 I.N. Stranski, Z. Phys. Chem. B 11, 342 (1931)

22 I.N. Stranski, R. Kaischew, Z. Kristallogr. 78, 373 (1931)

23 I.N. Stranski, R. Kaischew, Ann. Phys. 23, 330 (1935)

24 I.N. Stranski, R. Suhrmann, Z. Kristallogr. 105, 481 (1944)

25 I.N. Stranski, R. Suhrmann, Ann. Phys. 1, 153 (1947)

26 I.N. Stranski, Discuss. Faraday Soc. 5, 13 (1949)

27 C. Herring, Phys. Rev. 82, 87 (1951)

28 C. Herring, The Use of Classical Macroscopic Concepts in Surface Energy Problems, in Structure and Properties of Solid Surfaces, ed. by R. Gomer, C.S. Smith (University of Chicago, Chicago, 1952), p. 5

29 J. Friedel, B.D. Cullity, C. Crussard, Acta Metall. 1, 79 (1953)

30 J.K. MacKenzie, A.J.W. Moore, J.F. Nicholas, J. Phys. Chem. Solids 23, 185 (1962)

31 I.N. Stranski, Z. Phys. Chem. 136, 259 (1928)

32 W.K. Burton, N. Cabrera, Discuss. Faraday Soc. 5, 33 (1949)

33 W.K. Burton, N. Cabrera, F.C. Frank, Philos. Trans. R. Soc. London 243A, 299 (1951)

34 E.F. Gruber, W.W. Mullins, J. Phys. Chem. Solids 28, 875 (1967)

35 W.W. Mullins, Solid Surface Morphologies Governed by Capillarity, in Metal Surfaces: Structure, Energetics and Kinetics, ed. by W.D. Robertson, N.A. Gjostein (American Soc. for Metals, Metals Park, Ohio, USA, 1963), pp. 17-66

36 N. Cabrera, Surf. Sci. 2, 320 (1964)

37 C. Jayaprakash, C. Rottman, W.F. Saam, Phys. Rev. B 30, 6549 (1984)

38 C. Jayaprakash, W.F. Saam, Phys. Rev. B 30, 3916 (1984)

39 N. Garcia, P.A. Serena, Surf. Sci. 330, L665 (1995)

40 J.J. Sáenz, N. García, Surf. Sci. 155, 24 (1985) 
41 C. Rottman, M. Wortis, J.C. Heyraud, J.J. Métois, Phys. Rev. Lett. 52, 1009 (1984)

42 J.J. Métois, J.C. Heyraud, Surf. Sci. 180, 647 (1987)

43 M. Nowicki, C. Bombis, A. Emundts, H.P. Bonzel, P. Wynblatt, New J. Phys. 4, 60 (2002)

44 H.P. Bonzel, Phys. Rep. 385, 1 (2003)

45 G. Wulff, Z. Kristallogr. 34, 449 (1901)

46 A. Dinghas, Z. Kristallogr. 105, 304 (1944)

47 L.D. Landau, E.M. Lifshitz, Statistical Physics (Addison-Wesley, Reading, 1958), vol. V, p. 460

48 A.R. Roosen, R. McCormack, W.C. Carter, Wulff Construction Software: http://www.ctcms.nist.gov/wulffman/, National Institute of Standards and Technology (1999)

49 J.C. Heyraud, J.J. Métois, J. Cryst. Growth 50, 571 (1980)

50 J.C. Heyraud, J.J. Métois, Acta Metall. 28, 1789 (1980)

51 J.C. Heyraud, J.J. Métois, Surf. Sci. 128, 334 (1983)

52 J.J. Métois, G.D.T. Spiller, J.A. Venables, Philos. Mag. A 46, 1015 (1982)

53 H.P. Bonzel, A. Emundts, Phys. Rev. Lett. 84, 5804 (2000)

54 M. Wortis, in Chemistry and Physics of Solid Surfaces, ed. by R. Vanselow, R. Howe (Springer, New York, 1988), vol. 7, p. 367-405

55 D. Yu, H.P. Bonzel, M. Scheffler, New J. Phys. 8, 65 (2006)

56 P. Feibelman, Phys. Rev. B 65, 129902 (2002)

57 C.M. Wei, M.Y. Chou, Phys. Rev. B 66, 233408 (2002)

58 M. Fuchs, M. Scheffler, Comput. Phys. Commun. 119, 67 (1999)

59 S. Wei, M.Y. Chou, Phys. Rev. B 50, 4859 (1994)

60 C. Rottman, M. Wortis, Phys. Rev. B 29, 328 (1984)

61 R. Smoluchowski, Phys. Rev. 60, 661 (1941)

62 M.W. Finnis, V. Heine, J. Phys. F Met. Phys. 4, L37 (1974)

63 M.C. Desjonquères, D. Spanjaard, C. Barreteau, F. Raouafi, Phys. Rev. Lett. 88, 056104 (2002)

64 M. Nowicki, A. Emundts, H.P. Bonzel, Prog. Surf. Sci. 74, 123 (2003)

65 J.J. Métois, J.C. Heyraud, J. Cryst. Growth 57, 487 (1982)

66 G. Rao, D.B. Zhang, P. Wynblatt, Acta Metall. Mater. 41, 3331 (1993)

67 A. Emundts, P. Coenen, G. Pirug, B. Voigtländer, H.P. Bonzel, P. Wynblatt, Rev. Sci. Instrum. 72, 3546 (2001)

68 S. Surnev, P. Coenen, B. Voigtländer, H.P. Bonzel, P. Wynblatt, Phys. Rev. B 56, 12131 (1997)

69 S. Surnev, K. Arenhold, P. Coenen, B. Voigtländer, H.P. Bonzel, P. Wynblatt, J. Vac. Sci. Technol. A 16, 1059 (1998)

70 K. Arenhold, S. Surnev, H.P. Bonzel, P. Wynblatt, Surf. Sci. 424, 271 (1999)

71 H.P. Bonzel, Prog. Surf. Sci. 67, 45 (2001)

72 H.P. Bonzel, Interface Sci. 9, 21 (2001)

73 K. Thürmer, J.E. Reutt-Robey, E.D. Williams, M. Uwaha, A. Emundts, H.P. Bonzel, Phys. Rev. Lett. 87, 186102 (2001)
74 A. Emundts, M. Nowicki, H.P. Bonzel, Surf. Sci. 496, L35 L42 (2002)

75 M. Nowicki, C. Bombis, A. Emundts, H.P. Bonzel, P. Wynblatt, Eur. Phys. Lett. 59, 239 (2002)

76 M. Nowicki, C. Bombis, A. Emundts, H.P. Bonzel, Phys. Rev. B 67, 075405 (2003)

77 H.P. Bonzel, M. Nowicki, Phys. Rev. B 70, 245430 (2004)

78 W.W. Mullins, G.S. Rohrer, J. Am. Ceram. Soc. 83, 214 (2000)

79 G.S. Rohrer, C.L. Rohrer, W.W. Mullins, J. Am. Ceram. Soc. 84, 2099 (2001)

80 J.P. Perdew, J.A. Chevary, S.H. Vosko, K.A. Jackson, M.R. Pederson, D.J. Singh, C. Flolhais, Phys. Rev. B 46, 6671 (1992)

81 J.P. Perdew, K. Burke, M. Ernzerhof, Phys. Rev. Lett. 77, 3865 (1996)

82 V.N. Staroverov, G.E. Scuseria, J. Tao, J.P. Perdew, Phys. Rev. B 69, 075102 (2004)

83 V.K. Kumikov, K.B. Khokonov, J. Appl. Phys. 54, 1346 (1983)

84 D. Chatain, J.J. Métois, Surf. Sci. 291, 1 (1993)

85 D. Alfe, M.J. Gillan, J. Phys. C Condens. Matter 18, L435 (2006)

86 N. Akutsu, Y. Akutsu, J. Phys. C Condens. Matter 11, 6635 (1999)

87 C. Steimer, M. Giesen, L. Verheij, H. Ibach, Phys. Rev. B 64, 085416 (2001)

88 D.C. Schlösser, L.K. Verheij, G. Rosenfeld, G. Comsa, Phys. Rev. Lett. 82, 3843 (1999)

89 G. Schulze Icking-Konert, M. Giesen, H. Ibach, Phys. Rev. Lett. 83, 3880 (1999)

90 P. Feibelman, Phys. Rev. B 60, 11118 (1999)

91 A. Pavlovska, K. Faulian, E. Bauer, Surf. Sci. 221, 233 (1989)

92 A. Pavlovska, D. Dobrev, E. Bauer, Surf. Sci. 326, 101 (1995)

93 U. Gangopadhyay, P. Wynblatt, Met. Mater. Trans. A 25, 607 (1994)

94 J.C. Heyraud, J.J. Métois, J. Cryst. Growth 82, 269 (1987)

95 L. Kuipers, J.W.M. Frenken, Phys. Rev. Lett. 70, 3907 (1993)

96 L. Kuipers, M.S. Hoogeman, J.W.M. Frenken, Surf. Sci. 340, 231 (1995)

97 K. Thürmer, J.E. Reutt-Robey, E.D. Williams, Surf. Sci. 537, 123 (2003)

98 M. Ranganathan, D.B. Dougherty, W.G. Cullen, T. Zhao, J.D. Weeks, E.D. Williams, Phys. Rev. Lett. 95, 225505 (2005)

99 M. Degawa, W. Cullen, A. Pimpinelli, T.L. Einstein, E.D. Williams, Phys. Rev. Lett. 97, 080601 (2006)

100 N. Moll, A. Kley, E. Pehlke, M. Scheffler, Phys. Rev. B 54, 8844 (1996)

101 Q.K.K. Liu, N. Moll, M. Scheffler, E. Pehlke, Phys. Rev. B 60, 17008 (1999)

102 E. Pehlke, N. Moll, A. Kley, M. Scheffler, Appl. Phys. A 65, 525 (1997)

103 N. Moll, M. Scheffler, E. Pehlke, Phys. Rev. B 58, 4566 (1998)

104 M. Degawa, F. Szalma, E.D. Williams, Surf. Sci. 583, 126 (2005) 